\title{
ZONIFICACIÓN PAISAJÍSTICA PARA LA PLANIFICACIÓN TERRITORIAL: EL CASO DE LAS DIRECTRICES DEL BAJO ARAGÓN
}

\author{
Javier ALBISU, Juan DE LA RIVA, Mónica GARCÍA, Paloma IBARRA y Pedro SESÉ \\ Departamento de Geografía y Ordenación del Territorio \\ Universidad de Zaragoza
}

\begin{abstract}
Resumen: En el marco conceptual y metodológico del estudio geosistémico, se procede a la zonificación del territorio en unidades de paisaje a partir del análisis de sus componentes a diversas escalas. Para la definición de subunidades, el uso del suelo y los rasgos geomorfológicos se revelan de gran importancia. Ello colabora a la definición de un modelo territorial para la propuesta de directrices de ordenación de la comarca del Bajo Aragón.
\end{abstract}

Palabras clave: Paisaje, zonificación, análisis geosistémico, planificación, Bajo Aragón.

\begin{abstract}
The zonification of territory in landsacpe units was made in accordance to the geosistemic analysis framework by studing its components in different scales. Land uses and geomorphological features are two important characteristics in subunits definition. This settings served as the base to the territorial model definition of the Bajo Aragón region planning strategy.
\end{abstract}

Key words: Landscape, zonification, geosistemic analysis, planning, Bajo Aragón.

\section{INTRODUCCIÓN}

La Comunidad Autónoma aragonesa, en el ejercicio de sus competencias en la materia y según lo establecido por la Ley 11/1992 de Ordenación del Territorio de Aragón (BOA n ${ }^{\circ}$ 142, de 7 de diciembre), se ha dotado de un marco básico de planificación regional, plasmado en las Directrices de Ordenación Territorial de Aragón (Ley 7/1998, BOCA n 201, de 9 de julio). Junto a éstas - y para su desarrollo- la Ley 11/1992 previó la elaboración de Directrices Parciales de una doble naturaleza: de ámbito territorial y sectoriales. Aquellas 
de carácter territorial se refieren fundamentalmente a áreas homogéneas o funcionales, que se han hecho corresponder con las fijadas por el nuevo mapa de comarcas de Aragón, aprobado por Decreto Ley 8/1996, de 2 de diciembre, de Delimitación Comarcal; a estas últimas deberá someterse tanto el planeamiento municipal como la planificación sectorial.

El presente trabajo recoge algunos aspectos del análisis y diagnóstico desarrollados en el Proyecto de Directrices Parciales de Ordenación Territorial de la comarca 28, Bajo Aragón (V. Figura 1$)^{1}$. Entre las determinaciones que la Ley 11/1992 fija para esta figura de planeamiento (art. 26), los autores de este artículo atendieron especialmente al estudio relativo al medio natural y sus recursos, al modelo de ocupación del suelo, al señalamiento de áreas sometidas a algún tipo de protección y a la fijación de criterios para el uso y clasificación del territorio. En definitiva, aspectos diversos del análisis territorial en torno al segundo de los definidos por la Ley 11/1992 como elementos estructurantes, el medio natural, siendo los otros la población y su distribución, los sistemas generales (servicios, infraestructuras...) y los sistemas económicos.

En consecuencia, omitiendo otros aspectos de la Directriz Parcial propuesta para el territorio, nos centraremos aquí en el análisis sintético del medio natural y, más concretamente, del paisaje. Éste desempeña, en cuanto Sistema Natural, un papel articulador dentro del Modelo Territorial definido para la comarca, juntamente con otros elementos como las infraestructuras. Este hecho fundamental conduce a la necesidad de caracterizar y analizar en su valor y en su función articuladora el elemento «paisaje» desde la consideración de sus rasgos naturales; este papel se materializa en la comarca, fundamentalmente, en la existencia de unos sistemas fluviales que la recorren en sentido meridiano y en la destacada impronta de las Serranías Ibéricas, de dirección contrapuesta, que establecen así el contrapunto a la Tierra Baja más septentrional.

En el contexto de los objetivos globales perseguidos para la zona en la Directriz mantenimiento de la población, mejora de la calidad de vida de los habitantes y potenciación del desarrollo económico sostenible, mediante el uso racional del territorio y el respeto a sus valores naturales - se define uno más específico, relativo a la articulación de los Sistemas Naturales: salvaguardar y restaurar el medio ambiente natural, equipándolo para hacer posible su disfrute por la población e identificando aquellas áreas susceptibles de especial protección. En conexión con lo anterior, se persigue también la fijación de criterios de ordenación y aprovechamiento de usos del suelo acordes con las características naturales y paisajísticas del territorio. Todo ello implica la definición del papel estratégico del Sistema Natural, la sistematización de aquellos espacios con significación relevante y las

1 Dicho trabajo fue elaborado para el Departamento de Ordenación Territorial, Obras Públicas y Transportes del Gobierno de Aragón por el siguiente equipo multidisciplinar: P. de la Cal, G. Molpeceres, M. Abad, J.A. Alfaro, I. de Rosendo, G. Oliván, Arquitectos; J. de la Riva, P. Ibarra, J. Albisu, M. García, P. Sesé, Geógrafos; A. Latorre, Ingeniero Agrónomo; J.A. Navamuel, Ingeniero de Caminos.

Conforme a lo establecido en la Ley 11/92, el documento de la Directriz atiende a los siguientes aspectos: el análisis y diagnóstico de los problemas y oportunidades, las indicaciones para la comprensión del modelo territorial propuesto, el texto normativo propiamente dicho, el programa de actuación. 

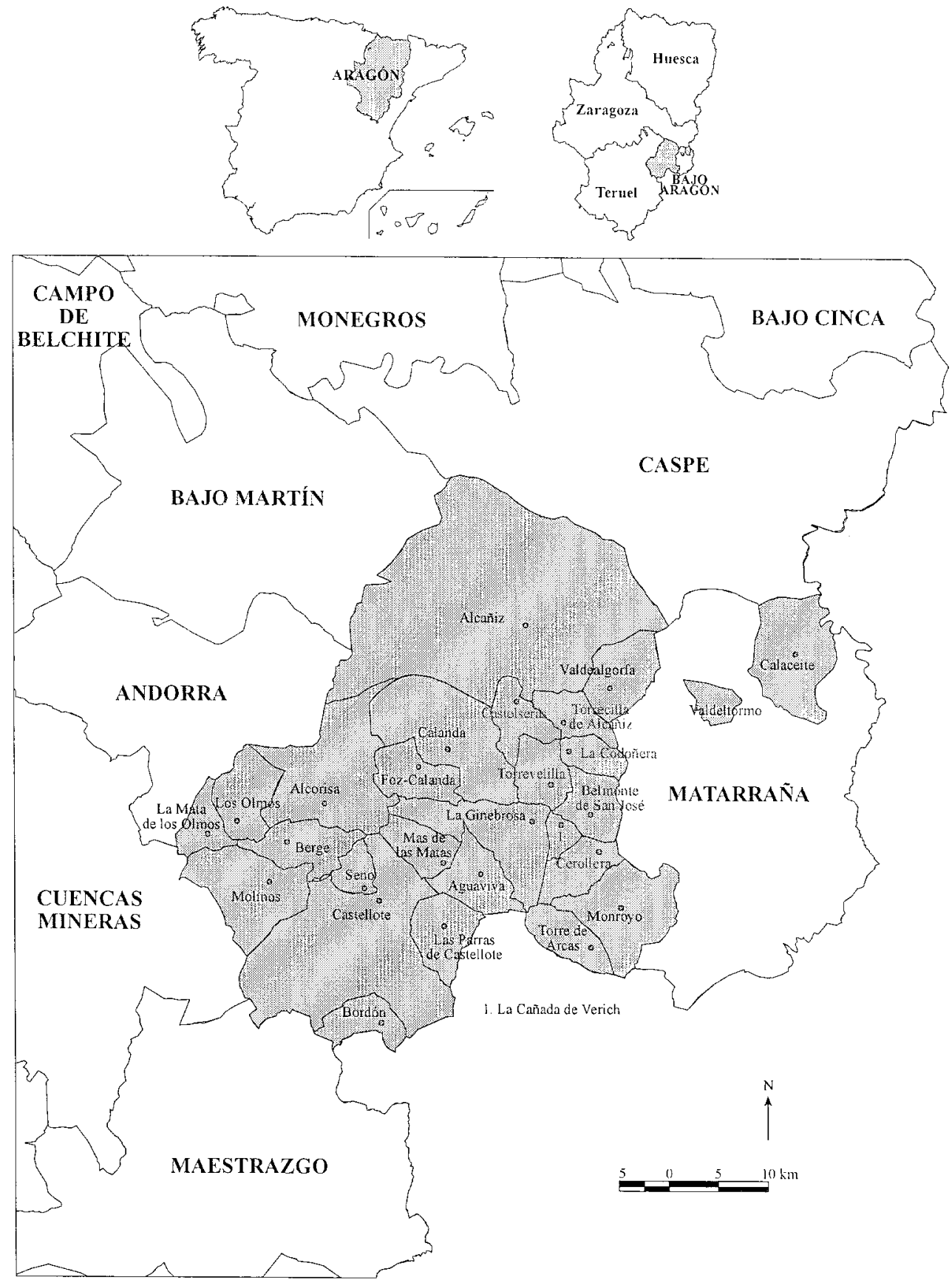

Figura 1. Área de estudio 
pertinentes recomendaciones para su uso y, finalmente, el establecimiento de pautas de asignación de funciones y definición de actuaciones.

\section{METODOLOGÍA}

En la anterior dirección se orienta el análisis y diagnóstico realizados a través de las unidades paisajísticas delimitadas. Queremos destacar, tal como se puso de manifiesto en el III Congreso de la Ciencia del Paisaje (Berga, 1999), que «en los últimos años ha ido fraguándose un cuerpo conceptual y metodológico capaz de sustentar el tratamiento científico del paisaje (...) al mismo tiempo que se ha producido un incremento considerable de la vertiente aplicada de estos estudios, en especial por los aspectos relacionados con la protección, la planificación y la gestión territorial» (PÉREZ CHACÓN, 1999). Y es que el paisaje, desde el punto de vista geosistémico, es un concepto integrador, acorde con los objetivos actuales de tratamiento global de los problemas territoriales. De acuerdo con esta línea de investigación aplicada, se presenta una síntesis del estudio de paisaje realizado en los apartados de análisis y diagnóstico, para esbozar después las directrices concretas que a aquél hacen referencia.

El método aplicado para abordar la zonificación del territorio en unidades de paisaje se enmarca en la concepción geosistémica del territorio y en los análisis de paisaje integrado (BOLÓS, 1992; IBARRA, 1993). De acuerdo con los principios metodológicos que guían estos estudios, se entiende por unidad de paisaje un espacio que, a una escala determinada, se caracteriza por una fisionomía homogénea y una evolución común, siendo de unas dimensiones concretas y cartografiables. En función de la escala y del consiguiente grado de homogeneidad, se pueden diferenciar varios niveles de unidades de paisajes que se ordenan jerárquicamente. Dados los objetivos y escala del presente trabajo se han delimitado unidades de paisaje a dos escalas: grandes unidades a escala IV (según la escala taxonómica de TRICART y CAILLEUX, 1956) con unas dimensiones que oscilan entre 100 $\mathrm{km}^{2}$ y $1.000 \mathrm{~km}^{2}$, y subunidades a escala $V$ (e incluso VI en algunos casos), con un nivel de homogeneidad mayor y de unas dimensiones que oscilan entre $10 \mathrm{~km}^{2}$ y $100 \mathrm{~km}^{2}$.

El proceso de delimitación de estas unidades de paisaje requiere, como etapas previas, junto a la recopilación de información, el análisis, la descripción y la plasmación cartográfica de los diferentes componentes del paisaje (relieve, litología, clima, suelos, vegetación y usos del suelo...). A partir de ahí, se aborda el diagnóstico sobre el peso de cada elemento analizado en la estructuración del paisaje a las escalas seleccionadas; ello permite fijar los criterios a utilizar para la delimitación de las mencionadas unidades de paisaje. A continuación se realiza la zonificación o delimitación de las diferentes unidades combinando la cartografía de los elementos seleccionados previamente como criterios. La primera zonificación resultante requiere una posterior campaña de trabajo de campo para comprobar in situ la adecuación de las unidades definidas y realizar las oportunas correcciones. Por 
último, tras la elaboración del definitivo mapa de paisaje, se realiza una breve descripción de cada una de las unidades, poniendo en relación la información de los diferentes elementos antes analizados aisladamente.

Los elementos del paisaje seleccionados como criterios de delimitación de las grandes unidades de paisaje en que se estructura la comarca del Bajo Aragón son, en primer lugar, el relieve, la geología, el clima y los suelos y, en segundo lugar, el agua. Así, se han diferenciado tres grandes zonas o unidades de paisaje: la Tierra Baja, las Serranías Ibéricas y las superficies dominadas por grandes láminas de agua (embalses). Estas unidades se han subdividido en diversas subunidades de paisaje en función, principalmente, de los usos del suelo (Figura 2), combinados con rasgos geomorfológicos de mayor detalle. La elaboración del mapa de usos del suelo es así una tarea tan imprescindible como laboriosa; el que aquí se presenta ha sido obtenido mediante fotointerpretación convencional, sobre la base de una leyenda jerárquica y adaptada a los requerimientos del análisis.

\section{UNIDADES DE PAISAJE DE LA COMARCA DEL BAJO ARAGÓN}

Se describen a continuación los principales rasgos de las unidades de paisaje delimitadas y que se plasman cartográficamente en la Figura 3. En cada caso se incluyen entre paréntesis las denominaciones según la toponimia local, a las que se hace referencia en las directrices.

\section{La Tierra Baja}

Ocupa un 45,22\% de la comarca y se extiende por la mitad septentrional, identificándose con el Valle del Ebro y con los glacis y sectores más bajos del piedemonte serrano. Es, en conjunto, una zona muy homogénea, de bajas pendientes y escasa compartimentación de terreno; ello da lugar a una intensa explotación agrícola. Este territorio puede subdividirse en las siguientes unidades de paisaje:

- Paisajes agrícolas de la Tierra Baja occidental (Secanos de Vizcuerno, Regadíos de Puigmoreno-Valmuel, Regadío tradicional de Alcañiz, Secano de Alcañiz, Secanos de Alcañiz-Calanda, Nuevos regadíos de Calanda-Alcañiz, Olivar de Calanda).

Son las zonas más llanas, con suelos desarrollados y aptos para la agricultura. En función de los usos dél suelo dominantes se diferencian las siguientes subunidades: Paisajes agrícolas de secano en áreas llanas, Paisajes agrícolas de regadío y Paisajes agrícolas de leñosos en el contacto con las Serranías Ibéricas.

La posibilidad de un óptimo aprovechamiento de los recursos hídricos mediante las infraestructuras creadas y la aplicación de las nuevas técnicas de rega- 







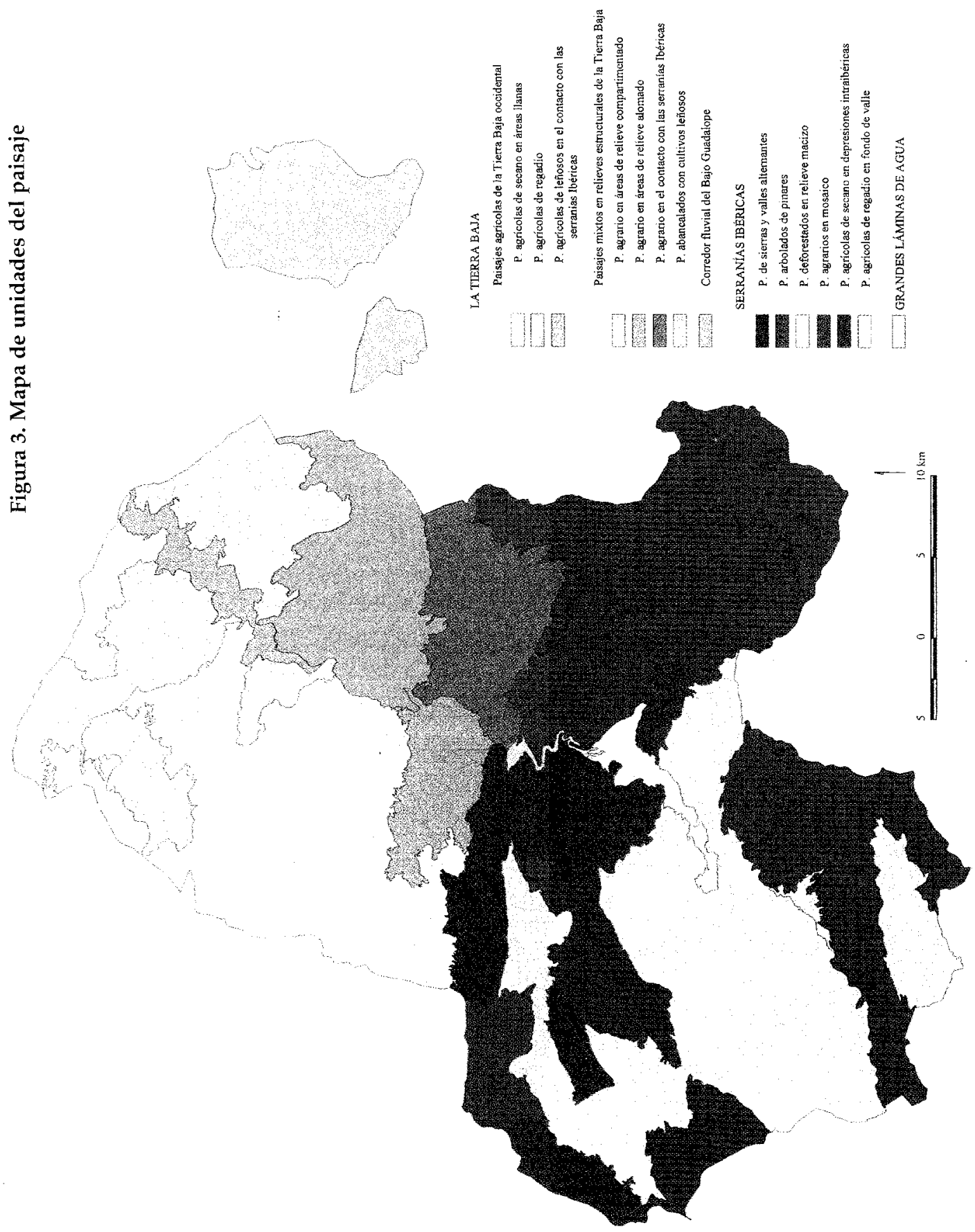


dío, unido a la planitud del terreno y a unos suelos adecuados, son elementos que posibilitan una agricultura industrial. La presencia de heladas tardías y la aridez del clima mediterráneo continentalizado, que puede acarrear períodos de escasez de agua y que condiciona el tipo de vegetación, con ausencia significativa de grandes extensiones naturales de bosques, son factores limitantes a considerar.

Este espacio incluye importantes enclaves naturales como las Saladas y las áreas endorreicas de Alcañiz, que constituyen un paisaje de interés científico, por cuanto albergan especies de alto valor ecológico y diversos endemismos, como Halopeplis amplexicaulis y Microcnemum coralloides, cuya subsistencia es muy vulnerable. Las áreas endorreicas son espacios de una gran fragilidad ecológica, por lo que la puesta en marcha de nuevos regadíos puede modificar de manera significativa el ecosistema de estos enclaves. Por otro lado, la presencia de relieves invertidos, paleocanales, con alto interés paisajístico, científico y educativo, es otro elemento que puede revitalizar las posibilidades económico-culturales de la unidad.

- Paisajes mixtos en relieves estructurales de la Tierra Baja (Paisajes agrarios de Alcañiz, de Castelserás y del área Mezquín-Guadalope, Paisajes abancalados de Valdeltormo y Calaceite).

Esta unidad ocupa la mitad oriental de las Tierras Bajas, extendiéndose hacia el noroeste por la Sierra del Vizcuerno. Se sitúa entre los 400 y los $600 \mathrm{~m}$ y presenta una topografía bastante compartimentada, donde se alternan las serrezuelas con los pequeños valles de fondo plano. En ella es posible distinguir las siguientes subunidades: Paisajes agrarios en áreas de relieve compartimentado, Paisajes agrarios en áreas de relieve alomado, Paisajes agrarios en el contacto con las Serranías Ibéricas y Paisajes abancalados con cultivos leñosos.

Desde el punto de vista agrícola, la compartimentación del terreno y el aprovechamiento de los espacios cultivables ha generado un profuso abancalamiento de laderas y vales, instalándose cultivos leñosos típicamente mediterráneos como el olivo y el almendro. Los cultivos tienen calidad suficiente para generar productos de alto valor añadido. Por otro lado, se produce una combinación paisajística de gran interés y belleza entre los espacios naturales y los cultivos leñosos en bancales.

El patrimonio natural de esta unidad presenta también potencialidades todavía no desarrolladas en su conjunto. Las características topográficas, que supusieron un condicionante importante para el desarrollo de actividades agrícolas, se convierten en rasgos positivos por las interesantes formaciones boscosas que propician y actualmente soportan. Por todo ello, estos espacios presentan una triple potencialidad: explotación forestal, recursos cinegéticos y florísticos, potencial turístico.

Los incendios forestales son un factor limitante de primer orden; junto a la evidente afección paisajística, la desaparición de las masas forestales desencadena toda 
una serie de procesos degradativos del medio natural (aceleración de la erosión, destrucción de hábitats faunísticos, pérdida de la capacidad de retención de agua), además de incidir negativamente aspectos socio-económicos como son la pérdida de espacios de ocio y esparcimiento, explotaciones forestales, etc.

- Corredor fluvial del Bajo Guadalope.

Se trata de una estrecha banda que transcurre semiparalela a los meandros que presenta el río Guadalope en este tramo (desde Castelserás, donde confluye con el río Mezquín, hasta su salida de la comarca). La mayor disponibilidad de agua y los suelos aluviales suponen la base idónea para el aprovechamiento de estas llanas y fértiles tierras con cultivos de regadío tradicionales. Reúne importantes valores naturales al ser un corredor fluvial con pequeños sotos que albergan flora y fauna valiosa y que se comunican con los espacios seminaturales de las serrezuelas colindantes; por otro lado, son de reseñar también sus interesantes meandros divagantes.

\section{Las Serranías Ibéricas}

La unidad de las Serranías Ibéricas ocupa un 54,78\% de la comarca (101.767 has). Comprende la zona central y meridional, abarcando aquellas zonas de las sierras (800-1200 m.) y depresiones intraibéricas $(500-700 \mathrm{~m}$.), de relieves más o menos compartimentados, donde las pendientes son superiores a las de la Tierra Baja y el porcentaje de tierras de dedicación agrícola es inferior. Allí se registran las temperaturas medias anuales más bajas de la comarca, especialmente en el extremo meridional, coincidiendo con las mayores altitudes; ello explica en parte la menor explotación agrícola del territorio y el mayor peso de los recursos forestales.

Dominan los paisajes más agrestes, de pendientes más fuertes y mayor altitud, siendo elementos paisajísticos típicos los pinares, los relieves redondeados sin apenas cobertura vegetal, o los mosaicos de explotación agrícola con bosque seminatural o de repoblación. En cuanto a los usos del suelo predominan las superficies cubiertas por bosques seminaturales y las zonas ocupadas por matorral y pastizal. Estas últimas, que suponen el $16 \%$, revelan un medio natural degradado, fruto de anteriores modos en el sistema de explotación de los recursos, pero manifiestan una gran capacidad de recuperación del medio como ecosistema natural.

En su conjunto, esta compartimentada y compleja unidad, de carácter fundamentalmente forestal, contiene numerosos aspectos con gran potencialidad. Los espacios agrícolas resultan aptos para el cultivo de leñosas, olivo y almendro, a pesar de los fenómenos atmosféricos adversos (heladas tardías, granizo, etc.). Las potencialidades naturales, que son las menos desarrolladas, residen en muy diversos elementos: abruptos relieves y áreas 
donde afloran materiales calizos en los que se generan procesos kársticos y cuevas de gran belleza, como las de Molinos; enclaves naturales singulares como foces, fallas, pliegues, gargantas del río Guadalope y elementos singulares como los árboles fósiles de Castellote; elementos florísticos protegidos y amenazados, como Thymus loscosii y Petrocptis pardoi, y faunísticos como la cabra montesa, el águila real, etc.

Así mismo, existen importantes extensiones boscosas, con buenas potencialidades paisajísticas y forestales. Los incendios forestales han afectado especialmente a este espacio, destacando sobremanera el que en 1994 arrasó 12.432 has, principalmente de los municipios de Castellote y Bordón.

Las subunidades de paisaje que se han diferenciado son las siguientes:

- Paisajes de sierras y valles alternantes (Peñas Blancas).

Se trata del espacio - 500 a $700 \mathrm{~m}$ - comprendido entre la depresión de Calanda y un sector del piedemonte de la Sierra de los Caballos. Los condicionantes topográficos y la compartimentación del relieve hacen de ella una zona en la que se combinan los usos agrícolas predominantemente de secano en los fondos de los valles, con las tierras ocupadas por pastizales-matorrales localizadas en las laderas y zonas más agrestes.

- Paisajes arbolados de pinares (Pinares de Monroyo-La Ginebrosa, Cabecera del Guadalopillo, Pinares de Castellote-Bordón y Cerros de Alcorisa).

Se localizan en el sector oriental de la comarca, destacando las extensiones de pinares. Allá donde éstos se combinan con cultivos como el olivo, la constante paisajística marca la posición del pinar en las zonas superiores de las laderas, como en los municipios de Valdeltormo y Calaceite. En estos pinares, mayoritariamente de repoblación, coexisten masas naturales y otras que tienden a su naturalización, desarrollando un interesante sotobosque que es refugio de variada fauna. En aquellas zonas donde el terreno se hace más llano, fondos de valles y partes bajas de laderas, se extienden los usos agrícolas, predominantemente de secano tanto herbáceo como leñoso.

- Paisajes deforestados en relieve macizo (Serranías de Molinos-Castellote, Serranía de Bordón).

Esta subunidad, de alrededor de 34.000 has, agrupa buena parte del sector sudoeste de la comarca. La dureza del terreno y las características climáticas y edafológicas han propiciado un desarrollo importante del bosque de coníferas, que el hombre ha explotado efectuando talas y entresacas en un tipo de explotación que en los últimos años no responde al equilibrio ecológico del medio dentro de unos límites sostenibles.

- Paisajes agrarios en mosaico (Paisajes agrarios de Monroyo, de la Ginebrosa, de las Parras de Castellote, de Berge-Alcorisa y de Los Olmos). 
Dentro de este paisaje $-7,76 \%$ de la comarca- se incluyen aquellos espacios correspondientes a zonas de pendiente moderada y cuya altitud media ronda los 700 $\mathrm{m}$, si bien varía desde los 500 hasta los $900 \mathrm{~m}$. Se trata de zonas morfológicamente complejas, con sistemas de laderas, suelos de distinta evolución, microclima variado en función de la situación altitudinal y la orientación, el paisaje aparece condicionado por la fuerte compartimentación en cuanto a la cobertura vegetal y agraria.

- Paisajes agrícolas de secano en depresiones intraibéricas (Depresión de Mas de las Matas, Depresión de Aguaviva, Paisajes agrícolas de la Mata y Cantera Saso).

Esta subunidad se extiende, con sus 8.000 has, a lo largo de una banda con dirección oeste-este desde Los Olmos hasta Mas de las Matas, con una altitud media entre 700 y $900 \mathrm{~m}$. La dominante paisajística es el secano, pero también tiene un peso considerable el matorral-pastizal que pone de manifiesto el abandono de anteriores usos ganaderos.

- Paisajes agrícolas de regadío en fondo de valle (Regadíos Guadalope-Bergantes).

Esta pequeña unidad corresponde con las tierras fértiles de los ríos Guadalope y Bergantes. Se sitúa al sur del Embalse de Calanda, incluyendo parte de las tierras del piedemonte de la Sierra de La Ginebrosa, donde el terreno se hace algo más duro respecto a las vegas. La dominante paisajística se establece en torno a la presencia del recurso agua, ya que la zona de interfluvio entre estos dos ríos reúne buena parte de las tierras dedicadas a usos agrícolas de regadío de la comarca. Las formaciones arbóreas de ribera constituyen también una superficie a destacar.

\section{Superficies dominadas por grandes láminas de agua}

Esta unidad ocupa únicamente el $0,41 \%$ de la comarca, pero posee entidad propia para ser diferenciada del resto. El factor determinante es el agua, variable física de gran importancia en este territorio, ya que se trata de un recurso escaso y altamente demandado, especialmente en el área definida por la unidad de las Tierras Bajas.

Por ello, y por la importante extensión relativa que ocupan las láminas de agua permanentes, como los embalses (Santolea, Calanda, La Estanca de Alcañiz, Gallipuén), se ha considerado pertinente destacar agrupadamente estos espacios, como un importante recurso potencial de cara al desarrollo de la comarca. Todo ello no sólo en el ámbito agrario sino también turístico, por las actividades recreativas y de ocio que acogen y por constituir espacios de gran riqueza faunística, como es el caso de Santolea, protegido como Zona de Especial Protección para las Aves (ZEPA). El mayor problema de estos espacios deriva de la contaminación de sus aguas, bien por vertidos, bien por los productos utilizados en la agricultura, abonos, etc. 


\section{DIRECTRICES REFERIDAS A LAS UNIDADES DE PAISAJE}

Descritas someramente las unidades de paisaje delimitadas, se comentan ahora las líneas maestras vertidas al respecto en el articulado del documento de Directrices. Entre aquellas relativas a Ordenación, Protección y Desarrollo del Sistema Natural, en el art. 35, se afirma que los recursos naturales de la comarca del Bajo Aragón constituyen elementos fundamentales para el mantenimiento de los procesos vitales y para el desarrollo económico. Todas las iniciativas y normas de planeamiento territorial, así como las actuaciones públicas y privadas que les afecten, velarán por su conservación y promoverán una utilización de los mismos que no supere la capacidad de renovación de los recursos. Se señala como uno de los elementos que estructuran el territorio a las unidades paisajísticas, si bien poseen una entidad física independiente de la delimitación administrativa - municipal y comarcal-y, en muchos casos, trascienden el ámbito administrativo de la comarca.

El art. 37 se refiere a la utilización de las unidades paisajísticas y en él se afirma que el entendimiento del paisaje comarcal a partir de ellas se considera un aspecto crucial para unificar los criterios sectoriales de carácter supramunicipal en la planificación. La vocación territorial de estas unidades deberá ser potenciada desde las administraciones públicas, entendiendo además que se trata de áreas de carácter supracomarcal y que pueden servir como referencia para políticas de coordinación en este ámbito superior. Por ello, se considera conveniente utilizar la delimitación de las unidades paisajísticas a la hora de acometer las medidas de gestión medioambiental y, entre otros, los siguientes trabajos: planes de gestión forestal, proyectos de concentración parcelaria y regulación urbanística de usos.

En el art. 38 se propone un Plan de Restauración de elementos y áreas paisajísticas degradadas, entendiendo como tales los vertederos y puntos de acumulación de basuras, las ruinas, algunas infraestructuras no integradas (canales, tuberías, tendidos eléctricos...), las superficies removidas, las extracciones mineras o de áridos y los encauzamientos, dando prioridad a las que se encuentran en los entornos más visitados y en los de mayor calidad paisajística. Se promocionará el desarrollo de actividades relacionadas con el medio natural que planteen una alternativa válida para la recuperación de estas áreas, tales como albergues, campos de golf, áreas de esparcimiento, campings, centros ecuestres, centros deportivos, etc. Este Plan de Restauración contemplará la actuación prioritaria en los siguientes enclaves: vertedero en Castelserás, mina María Luisa en Castellote, antiguo vertedero de Mas de las Matas y tratamiento paisajístico en taludes y bordes de la carretera N-232 (tramo Las Ventas de Valdealgorfa-límite provincial).

El art. 39. se refiere a la integración paisajística de los núcleos de población, proponiendo que el planeamiento municipal propicie una estructura urbana adecuada para ello en relación con el medio físico que los rodea, estableciendo una definición cualificada de las características de los bordes urbanos, de su silueta y de los puntos de conexión con las infraestructuras de comunicación. El planeamiento municipal definirá así unas condiciones edificatorias que, desde el punto de vista estético y tipológico, resulten integradas en las 
características morfológicas de cada núcleo. Se prestará un especial cuidado en la regulación de los espacios públicos, cuidando las perspectivas paisajísticas de los núcleos urbanos.

El art. 40 se refiere al Análisis de Impacto Territorial e indica que será necesario realizarlo para cualquier obra, instalación o actividad autorizable que se pretenda realizar en los siguientes ámbitos de la comarca del Bajo Aragón:

- Espacios de interés natural que han sido propuestos por la Directriz para su inclusión en el Catálogo de Espacios Naturales Protegidos.

- Espacios de interés natural que han sido propuestos por la Directriz para su inclusión en el Catálogo de Areas Naturales Singulares pertenecientes al sistema de espacios forestales comarcales.

- Espacios que han sido identificados en la Directriz como Enclaves y Elementos Naturales Singulares.

- Entornos de núcleos de población identificados en la Directriz como Conjuntos Históricos de interés arquitectónico o ambiental.

Así mismo, los Análisis de Impacto Territorial serán exigibles de modo preventivo para cualquier uso, instalación o actividad en los siguientes casos: embalses, presas o pantanos; desarrollos urbanísticos de uso industrial con más de 4 has; desarrollos urbanísticos de uso residencial con más de 300 viviendas o con más de 5 has de superficie bruta; complejos turísticos con más de 300 camas; carreteras de nuevo trazado; grandes superficies comerciales, según la definición dada por la Ley 9/1989 sobre Equipamiento Comercial y Áreas de Esparcimiento.

Más adelante, al tratar en el articulado de la Directriz la Zonificación del Territorio (art. 77), se define como Suelo de Interés Paisajístico el formado por aquellos terrenos pertenecientes a las unidades de paisaje de mayor valor en la comarca: Peñas Blancas, Pinares de Monroyo-La Ginebrosa, Cabecera del Guadalopillo, Pinares de Castellote-Bordón, Cerros de Alcorisa, Serranías de Molinos-Castellote, Serranía de Bordón, Regadíos GuadalopeBergantes y Corredor del Bajo Guadalope.

En cuanto al Régimen de Protección se plantea la posibilidad de autorización de la explotación minera, la extracción de gravas y arenas, las canteras y la apertura de pistas y caminos; el resto de actividades no constructivas quedan permitidas. En cuanto a estas últimas, se permiten las construcciones e instalaciones de apoyo a la horticultura, los viveros e invernaderos, las construcciones destinadas a la ganadería extensiva, los corrales domésticos y las instalaciones apícolas; serán susceptibles de autorización las construcciones e instalaciones destinadas exclusivamente a la horticultura de ocio - sin que puedan destinarse a usos residenciales-, las construcciones e instalaciones aisladas destinadas a la ganadería intensiva, los almacenes agrícolas, las granjas, etc. 


\section{CONCLUSIÓN}

Del trabajo realizado se desprende la evidencia de la necesidad y utilidad, para una planificación territorial integrada, del estudio del sistema natural y, en especial, de los usos del suelo, como base para la definición de unidades de paisaje. Éstas pueden constituir así ámbitos espaciales de referencia para la aplicación de estrategias y actuaciones. En el caso del Bajo Aragón se ha llegado a una zonificación jerárquica que ha permitido la definición de ciertas propuestas, recogidas en la directrices apuntadas. No obstante, el análisis paisajístico desde la perspectiva geosistémica - tal y como se ha llevado a cabo- podría posibilitar un análisis integrado de otros aspectos de gran trascendencia paisajística, en especial lo relativo al sistema económico agrario.

\section{BIBLIOGRAFIA}

BOLÓS, M." (dir.) (1992): Manual de la Ciencia del Paisaje. Teoría, métodos y aplicaciones. Masson. Barcelona.

IBARRA, P. (1993): «Una propuesta metodológica para el estudio del paisaje integrado». Geographicalia 30, 229-242. Zaragoza.

PÉREZ-CHACÓN, E. (1999): «Líneas metodológicas en los estudios de paisaje». Ponencias III Congreso de Ciencia del Paisaje, 65-102. Universitat de Barcelona.

TRICART, J; CAILLEUX, A. (1956): «Le problème de la classification des faits géomorphologiques». Annales de Géographie LXV, 162-186. 